\title{
A REINFORCEMENT LEARNING APPROACH FOR MULTIAGENT NAVIGATION
}

\author{
Francisco Martinez-Gil, Fernando Barber, Miguel Lozano, Francisco Grimaldo \\ Departament d'Informatica, Universitat de Valencia, Campus de Burjassot, Burjassot, Valencia, Spain \\ Francisco.Martinez-Gil@uv.es, Fernando.Barber@uv.es,Miguel.Lozano@uv.es,Francisco.Grimaldo@uv.es \\ Fernando Fernández \\ Computer Science Department, Universidad Carlos III de Madrid, Leganés, Spain \\ ffernand@inf.uc3m.es
}

Keywords: Reinforcement learning. Multiagent systems. Local navigation.

\begin{abstract}
This paper presents a Q-Learning-based multiagent system oriented to provide navigation skills to simulation agents in virtual environments. We focus on learning local navigation behaviours from the interactions with other agents and the environment. We adopt an environment-independent state space representation to provide the required scalability of such kind of systems. In this way, we evaluate whether the learned action-value functions can be transferred to other agents to increase the size of the group without loosing behavioural quality. We explain the learning process defined and the results of the collective behaviours obtained in a well-known experiment in multiagent navigation: evacuation through a door.
\end{abstract}

\section{INTRODUCTION}

During last years, the most popular approaches to multiagent navigation have been inspired in different kind of rules (physical, social, etological, etc). These systems have demostrated that it is possible to group and to combine different rules (eg. cohesion, obstacle avoidance (Reynolds, 1987)) to finally display high quality collective navigational behaviours (eg. flocking). However the main drawbacks are also known. All the rules must be defined and adjusted manually by the engineer or author. The number of rules required for modelling complex autonomous behaviours can be high, and generally they are systems difficult to adjust when scaling the number of agents, where some problems (eg. local minimum or deadlocks) can appear. Beyond handwritten rules systems, other discrete techniques, as celular automata or multi-layer grids have been also used in these domains (Lozano et al., 2008) for representing different kind of navigational maps, as they can precompute important information for the agents when they have to plan and to follow their paths.

In this paper we present a Reinforcement Learning (RL) approach that consists on modelling the problem as a sequential decision problem using Markov Decision Process (MDP). Reinforcement learning tech- niques have been used successfully to find policies for local motion behaviors without knowledge of the environment (Kaelbling et al., 1996). It has been also applied in cooperative tasks, where several agents maximizes the collective performance by maximizing their individual rewards (Fernández et al., 2005). In these cooperative tasks, like Keepaway (Stone et al., 2005), the relationship among individual rewards and the cooperative behavior is typically unknown, but colaboration emerges from the individual behaviors.

The aim of the paper can be summarized in: a) setting a RL multiagent local navigation problem to simulate a single-door evacuation and b) to study the possibility of transferring the learned behaviors from a specific scenario to other bigger and more populated environments, which represents the basic concept of scalability in this domain. We propose the use of multiagent reinforcement learning using independent learners to avoid the "curse of dimensionality" of pure multiagent systems. We do not model the problem as a single-agent RL problem to allow the emergence of different solutions providing variability to the simulation. We explore the scalability (to hundreds of agents) and portability (to larger grids) of the approach. Scalability from a reduced set of agents to large ones is performed through the transfer of the value functions (Taylor and Stone, 2005). We 
show that the value functions (and hence, the policies) learned in scenarios with a few agents can be used in scenarios that multiplies the number of agents.

The paper has been organized as follows, section 2 describes the learning process used. In the section 3 we explain the motivation and use of the value function transfer. The section 4 shows the simulation results. The section 5 presents the main conclusions.

\section{Crowd Navigation as a RL Domain}

As independent learners, each agent's learning process can be modeled as a single-agent MDP. A MDP (Howard, 1960) is defined as a set of states $S$, a set of actions $A$, a stochastic transition function $T: S \times A \times S \rightarrow \Re$ and the reward function $\mathcal{R}$ : $S \times A \rightarrow \Re$ that specifies the agent's task. The agent's objective is to find an optimal policy, that is, a mapping from states to actions so as to maximize the expected sum of discounted reward, $E\left\{\sum_{j=0}^{\infty} \gamma^{j} r_{t+j}\right\}$ where $r_{t+j}$ is the reward received $j$ steps into the future. The discount factor $0<\gamma<1$ sets the influence of the future rewards. The optimal action-value function $Q(s, a)^{*}$ stores this expected value for each pair state-action in a discrete MDP. The Bellman optimality equation gives a recursive definition of the optimal action-value function $Q^{*}(s, a)=\mathcal{R}(s, a)+$ $\gamma \sum_{s^{\prime}} T\left(s, a, s^{\prime}\right) \max _{a^{\prime} \in A} Q^{*}\left(s^{\prime}, a^{\prime}\right)$. Usually the transition function $T$ is unknown, then the optimal policy can be learned through experience. There are several model-free algorithms to find $Q^{*}(s, a)$. In Q-learning algorithm (Watkins and Dayan, 1992) the agent starts with arbitrary values for the action-value function $\mathrm{Q}$, and updates the entry correspondent to time $t\left(s_{t}, a\right)$ from the new state $s_{t+1}$ receiving an inmediate reward $r_{t}$ as follows : $Q\left(s_{t}, a\right)=\left(1-\alpha_{t}\right) Q\left(s_{t}, a\right)+\alpha_{t}\left(r_{t}+\right.$ $\left.\gamma \max _{a^{\prime} \in A} Q\left(s_{t+1}, a^{\prime}\right)\right)$. This sequence converges to $Q^{*}(s, a)$ when all states are visited infinitely often and the learning rate $\alpha(t)$ has a bounded sum of its cuadratic value when $t \rightarrow \infty$.

In this work we present an experiment consisting on a group of agents that has to leave a region of the space reaching a door placed in the middle of a wall. This problem has two slopes. Individually, each agent has to learn to avoid other agents, avoid to crash against the wall and to learn the existing bias between the different actions in terms of effectiveness. As a group, the agents have to learn to cross the door in an organized way.

The features that describe the state are: a) one feature for the distance from the agent to the goal, b) eight features for the occupancy states of the eight neighbour positions c) one feature for the orientation

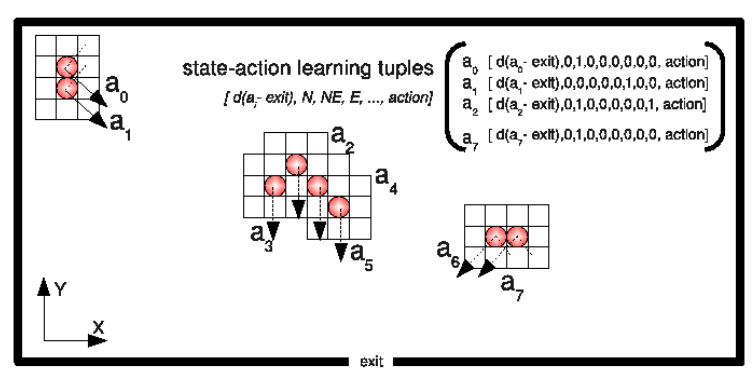

Figure 1: Multiagent learning situation example.

respect to the goal. There is no reference to position in the grid to allow portability. We have considered the Chesvichev distance because it is adequate to be used with diagonal movements. The set of actions consists on the eight possible unitary movements to the neighbor cells of the grid plus the action "Stay in your place". The agent is always oriented to the goal. The state configuration and the actions are relative to this orientation. For instance, in Figure 1, the arrows represent the same action ("go to the North") for the different agents and all the agents sensorize the north point in different places of the neighborhood.

We use Q-Learning with an $\varepsilon$-greedy exploratory policy and exploring starts because it is a simple and well-known model-free algorithm to converge to a stationary deterministic optimal policy of an MDP. The values of the learning algorithm are: the constant step-size parameter $\alpha=0.3$, the exploration parameter $\varepsilon=0.2$ with an exponential decay and a discount factor $\gamma=0.9$. The algorithm stops when an empirical maximum of trials is reached. If the agent reaches the goal, the reward value is 1.0; if the agent crash against a wall or with another agent, its immediate reward is -2.0 ; if the agent consumes the maximum allowed number of steps or cross the grid limits it is rewarded with a value of 0 . The immediate reward is always 0 for the rest of the middle states of a trial. The actionvalue functions are initialized optimistically to ensure that all the actions are explored. We have designed the described learning problem with 20 agents, therefore there are 20 independent learning processes.

The left curve of the Figure 2 shows the incremental mean of the expression $R=R_{F} \gamma^{t}$, where $R_{F}=$ $\{0,1\}, \gamma$ is the discount factor and $t$ is the lenght of the episode in steps. Besides the mean reward, the curve indicates a mean for the length of an episode in the interval $[7.0,8.0]$ that is coherent with the dimensions of the learning grid. The length of an episode is the number of decisions taken in this episode. The right curve displays the averaged lenght of the episodes. 

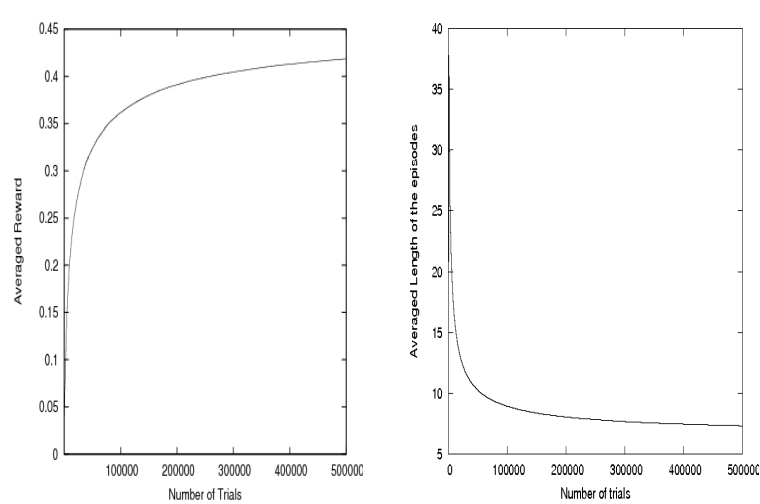

Figure 2: Mean reward curve and mean length of the episodes curve for one agent in the learning process.

\section{Scaling up the number of agents}

In simulation, we will use the learned value functions in a larger environment with different number of agents. We exploit the action-value functions using the greedy action selection as the optimal policy for an agent. Since the action and the state spaces are the same that used for learning, no mapping is required. However a generalization of the distance is necessary because the new grids are bigger in simulation time and agents can be placed initially farther than the learned distances. Our generalization criteria is based on the idea that the distance feature loses its discriminatory power when it is large. Therefore the distances higher than the MaxDist -1 value are mapped to a distance in the range [MaxDist - 1,MaxDist /2] using an empirical criteria.

Each action-value function is used to animate an incremental number of agents in the simulation environment to know their scalability. Thus then, the number of simulated agents grows in a factor $\times 1, \times 2$, $\times 3, \ldots, \times 10$ with the following meaning: a set of 20 functions corresponding with the learning process of 20 agents will have a scaling sequence of $\times 1=20$ agents, $\times 2=40$ agents, $\times 3=60$ agents, etc.

\section{Evaluating the Experiment}

We have defined five evaluation parameters.

1. Parameter 1 . It is the mean of random actions carried out by an agent and it is related with the quality of the learned action-value function. When the generalization process described formerly fails, the agent chooses a random action.

2. Parameter 2. It is the total number of crashes that happened in the simulation time. Bad learning of

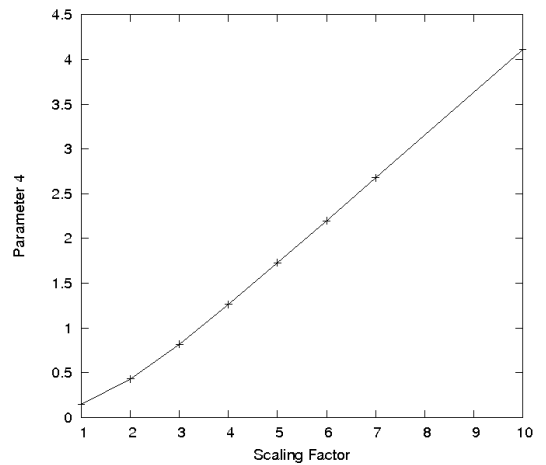

Figure 3: Parameter 4 for the experiment. These data are averages over 100 trials

states is a possible situation because convergence is warranted only with infinite iterations and to visit infinitelly often all the states is not guaranteed due to the on-line data adquisition based in the interaction with the environment.

3. Parameter 3. It is the total number of simulated episodes that have ended without success. When the agent has spent a maximum number of steps in one episode, it finishes with no success.

4. Parameter 4. It is the relative difference between the minimum amount of steps necessary to arrive to the exit from the initial position and the actual number of steps used. It represents the value $\left(\frac{l_{a c t}-l_{\min }}{l_{\min }}\right)$ where $l_{\min }$ is the minimum number of actions to reach the exit from a position with a single agent and $l_{\text {act }}$ is the number of actions actually carried out. It gives the idea of the difference between the actual performance and the minimum possible number of actions to reach the door. A value of 1.0 means that the number of actions is two times the minimum.

5. Parameter 5. It is an average density map that let us to estimate the collective behaviour achieved by the multiagent system during simulation. It gives a shape of the crowd in the grid, that is a reference parameter normally considered in pedestrian dynamic simulations (Helbing et al., 2000).

We have performed scaling simulations up to a scaling factor of $\times 10$ in the number of agents, corresponding to a maximum of 200 agents.

Concerning the Parameter 1, the percentage of aleatory actions used in simulation is $0 \%$ for our experiment in all the scaling factors. This result shows two facts: the generalization strategy has provided candidates in all the cases and the $\mathrm{Q}$ function for states near the goals have been learned to provide an answer to every query. Concerning the Parameter 2, the 


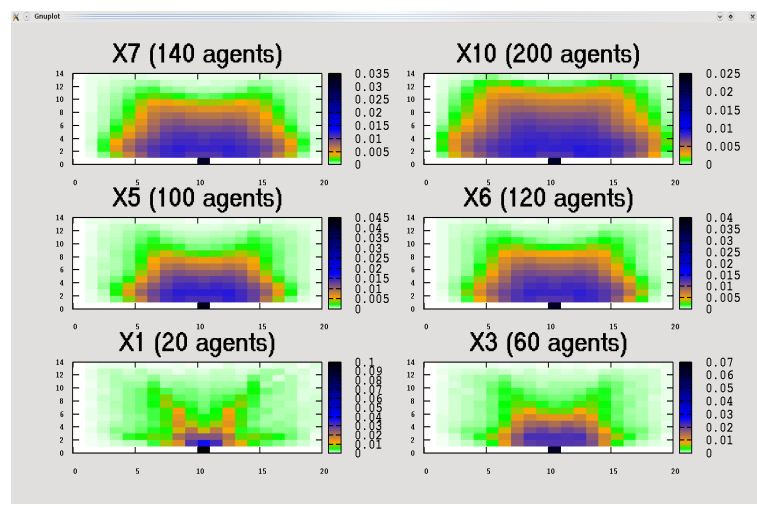

Figure 4: Average density map. The colors show densities, from low to high: white, green, orange, blue and black. Note the wall at the bottom in white with the door in black.

number of crashes against another agent or an obstacle are also 0 in all the scaling factors. It means that the agents have learned correctly to avoid crashes in all the encountered states. The Parameter 3 hints that all agents reach to the door. The results of these three parameters suggest that the transference of the learned value functions to the new simulation scenarios is adequate. The Figure 3 displays the results for Parameter 4 . In this curve, a value of 1.0 stands for an episode twice longer than the episode carried out by a single agent from the same initial position. The curve shows a lineal growth of the length of the episode with the scaling process. This lineal behavior allows us to predict the performance of the simulation in respect of the number of simulated agents.

The Figure 4 shows the average density map obtained for our experiment. It shows how the agents are gathering around the door in a typical half-circle shape as described in (Schadschneider A., 2009). We scale from 20 agents $(\times 1)$ up to $200(\times 10)$. In all the cases, the resulting shapings have been checked in the simulator (see Figure 5), giving good visual results.

\section{Conclusions}

Our results show that RL techniques can be useful to improve the scalability in the problem of controlling the navigation of crowded-oriented agents. In our experiment up to 200 agents are managed with a good behavior (a coherent decition sequence) using 20 learned value functions. Visual checking of the simulations agree with the experimental curves.

The relative sensorization and the independence of the cartesian position makes possible the scalability of the learned process to scenarios with different sizes

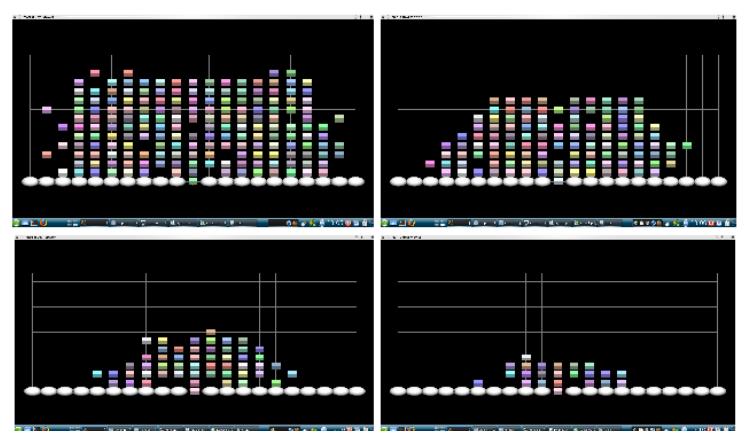

Figure 5: Different moments in simulation time in a $\times 10$ scale (200 agents). The agents are the square objects. The elipses build the wall. The door is placed at the centre of it.

and different position of the goals.

\section{Acknowledgements}

This work has been jointly supported by the Spanish MEC and the European Commission FEDER funds, under grants Consolider-Ingenio 2010 CSD2006-00046 and TIN2009-14475-C04-04.

\section{REFERENCES}

Fernández, F., Borrajo, D., and Parker, L. (2005). A reinforcement learning algorithm in cooperative multirobot domains. Journal of Intelligent Robotics Systems, 43(2-4):161-174.

Helbing, D., Farkas, I., and Vicsek, T. (2000). Simulating dynamical features of escape panic. Nature, 407:487.

Howard, R. A. (1960). Dynamic Programming and Markov Processes. The MIT Press.

Kaelbling, L. P., Littman, M. L., and Moore, A. W. (1996). Reinforcement learning: A survey. Int. Journal of Artificial Intelligence Research, 4:237-285.

Lozano, M., Morillo, P., Orduńa, J. M., Cavero, V., and Vigueras, G. (2008). A new system architecture for crowd simulation. J. Networks and Comp. App.

Reynolds, C. W. (1987). Flocks, herds and schools: A distributed behavioral model. In SIGGRAPH '87, pages 25-34, New York, NY, USA. ACM.

Schadschneider A., e. a. (2009). Encyclopedia of complexity and system science, chapter Evacuation dynamics: empirical results, modeling and applications, pages 3143-3166. Springer.

Stone, P., Sutton, R. S., and Kuhlmann, G. (2005). Reinforcement learning for RoboCup-soccer keepaway. Adaptive Behavior, 13(3). 
Taylor, M. E. and Stone, P. (2005). Behavior transfer for value-function-based reinforcement learning. In 4 th I.J.C. Autonomous Agents and Multiagent Systems.

Watkins, C. and Dayan, P. (1992). Q-learning. Machine Learning, 8:279-292. 